\title{
Determinants of prognosis of COPD in the elderly: mucus hypersecretion, infections, cardiovascular comorbidity
}

\author{
R. Pistelli*, P. Lange ${ }^{\#, \uparrow}$, D.L. Miller
}

Determinants of prognosis of COPD in the elderly: mucus hypersecretion, infections, cardiovascular comorbidity. R. Pistelli, P. Lange, D. L. Miller. (C) ERS Journals Ltd 2003. ABSTRACT: In this paper, the authors update the present knowledge about three risk factors for the prognosis of chronic obstructive pulmonary disease (COPD), which may be particularly relevant in elderly people: mucus hypersecretion, respiratory infections, and cardiovascular comorbidity. Chronic mucus hypersecretion (CMH) is a common respiratory symptom in old age, the relevance of which is analysed on the basis of data collected during the first three rounds of the Copenhagen City Heart Study. In subjects aged $\geqslant 65 \mathrm{yrs}, \mathrm{CMH}$ was a strong predictor of the incidence of respiratory infections in a 10-yr follow-up period and it was also a strong predictor of death from COPD (relative risk=2.5). However, $\mathrm{CMH}$ was associated with consistently lower forced expiratory volume in one second (FEV1) values, but not with an accelerated decline of FEV1 in this sample of an elderly population.

Acute respiratory infections (ARI) are extremely common at all ages, mostly mild self-limiting illnesses at a young age, but severe often fatal illnesses in elderly people already affected by a chronic disease such as COPD. This paper summarises the present knowledge about aetiology, pathology, prognostic relevance, and prevention of ARI. Furthermore, the areas in which further research is needed are listed. Clinical cohort studies clearly support the relevance of cardiovascular comorbidity for the shortand long-term prognosis of elderly subjects affected by severe COPD.

In this paper, the recently demonstrated association between particulate air pollution and cardiovascular events is reported to suggest the presence of an extremely susceptible cluster of elderly subjects in the population identified by the copresence of chronic obstructive pulmonary disease and cardiovascular comorbidity.

Eur Respir J 2003; 21: Suppl. 40, 10s-14s.
*Dept of Respiratory Physiology, Catholic University, Rome, Italy, ${ }^{\sharp}$ Copenhagen City Heart Study, Bispebjerg University Hospital, Copenhagen, Denmark, ${ }^{\circ}$ Dept of Respiratory Medicine, Hvidovre University Hospital, Hvidovre, Denmark, ${ }^{+}$Dept of Public Health Sciences, King's College, University of London, London, UK.

Correspondence: R. Pistelli

Dept of Respiratory Physiology

Catholic University

Via Moscati, 31

00168 Rome

Italy

Fax: 39063054641

E-mail: columbus.fpr@linet.it

Keywords: Cardiovascular comorbidity elderly

mucus hypersecretion

respiratory infections

Received and accepted: April 122002

\section{Chronic mucus hypersecretion}

Chronic mucus hypersecretion (CMH), defined as coughing and bringing up sputum for $>3$ months for at least two consecutive years, is a common respiratory symptom in elderly persons [1].

In the present analyses, the authors focused on the prevalence, possible risk factors for, and the prognostic importance of $\mathrm{CMH}$ in a random sample of an elderly population. Data were used from the first, second and third examination rounds of the Copenhagen City Heart Study, comprising 3,677 males and females aged $>65$ yrs, living in the inner city of Copenhagen. The original population sample was drawn in January 1976 from the Copenhagen Population Register among a population of $\sim 90,000$ inhabitants aged $\geqslant 20$ yrs [2]. The sample was age stratified, with the main emphasis placed on the age groups 35-70 yrs. The cohort was investigated for the first time in 1976-1978, for the second time in 1981-1983, and for the third time in 1991-1994. In general, the response rates were $\sim 65 \%$ in the participants $>65 \mathrm{yrs}$, being highest among subjects aged 65-70 yrs (75\%) and lowest among those aged $\geqslant 80$ yrs $(\sim 32 \%)$.

\section{Prevalence of and risk factors for chronic mucus hypersecretion}

In previous investigations of this cohort, the prevalence of $\mathrm{CMH}$ was $8.2 \%$ in females and $12.5 \%$ in males [3].
In subjects aged $>65$ yrs, the prevalence was somewhat higher, $13 \%$ in females and $18.6 \%$ in males. In both sexes, the prevalence was significantly related to smoking $(\mathrm{p}<0.001)$. For example, in never-smoking males the prevalence was $6.7 \%$, whereas in heavy-smoking males it was $23.4 \%$. Passive smoking, defined as being a nonsmoker and living with a smoking spouse, was associated with a significantly higher prevalence of $\mathrm{CMH}$ in females $(8.8 \%$ versus $12.6 \% ; \mathrm{p}<0.05)$, but not in males $(14.6 \%$ versus $11.4 \%)$.

The participants were subdivided into eight groups according to social status, mainly based on their education and type of work. In elderly females, there was no relationship between social status and prevalence of $\mathrm{CMH}$, whereas elderly males with low social status had a slightly higher prevalence of CMH $(p<0.05)$. The relatively low impact of social status on $\mathrm{CMH}$ in this elderly population is in contrast with findings relating to younger subjects in the Copenhagen City Heart Study [3]. This discrepancy probably reflects cohort differences in smoking habits; in the younger age groups, smoking is more prevalent in low social classes, whereas no social gradient in smoking habits is observed in the elderly sample. In addition, there are probably cohort differences with regard to occupation conditions. Previous exposure to dusts and fumes was significantly $(\mathrm{p}<0.001)$ related to higher CMH prevalence: $11.6 \%$ in females without exposure versus $28.4 \%$ in females with exposure, and in males $15 \%$ versus $27.7 \%$.

There was also a significant association between self-reported 
Table 1.-Results from multiple logistic regression predicting chronic mucus hypersecretion

\begin{tabular}{lc}
\hline Variable & OR $(95 \% \mathrm{CI})$ \\
\hline Male sex & $1.1(0.9-1.3)$ \\
Smoking & 1 \\
$\quad$ Never & $1.7(1.2-2.2)$ \\
$\quad$ Previous smoking & $2.8(2.1-3.8)$ \\
Present smoking & 1 \\
Multiple chest infections & $6.2(4.1-9.2)$ \\
$\quad<6$ in 10 yrs & $2.2(1.7-2.7)$ \\
$>6$ in 10 yrs & $2.1(1.6-2.9)$ \\
Dust and fumes at work & $1.8(1.3-2.3)$ \\
Chest infections in childhood & \\
\hline 3 drinks'day &
\end{tabular}

chest infections during childhood and CMH in old age: $12.1 \%$ versus $25 \%$ in females and $17.1 \%$ versus $40.6 \%$ in males. In addition, $\mathrm{CMH}$ was significantly more common in individuals with repeated chest infections during the 10 yrs before the $\mathrm{CMH}$ assessment. $\mathrm{CMH}$ was also more prevalent in individuals with a high consumption of alcohol.

A multiple logistic regression was performed, with $\mathrm{CMH}$ as the dependent variable and sex, active and passive smoking, childhood and recent respiratory infections, industrial dusts and fumes exposure, alcohol consumption and social status as independent variables. The regression showed that smoking, chest infections in childhood, recent chest infections and alcohol consumption of more than three drinks a day are significant predictors of CMH (table 1).

\section{Prognostic importance of chronic mucus hypersecretion}

The prognostic importance of $\mathrm{CMH}$ was investigated prospectively in different ways. Firstly, the incidence of respiratory infections during a 10 -yr follow-up was studied. The distribution of subjects according to presence of $\mathrm{CMH}$ and number of self-reported chest infections is shown in figure 1. This confirms that in this elderly population the presence of $\mathrm{CMH}$ is strongly related to respiratory infections.

In individuals with repeated measurements of forced expiratory volume in one second (FEV1) during the 15-yr observation period, the relation between lung function and $\mathrm{CMH}$ was investigated. Although the FEV1 was consistently

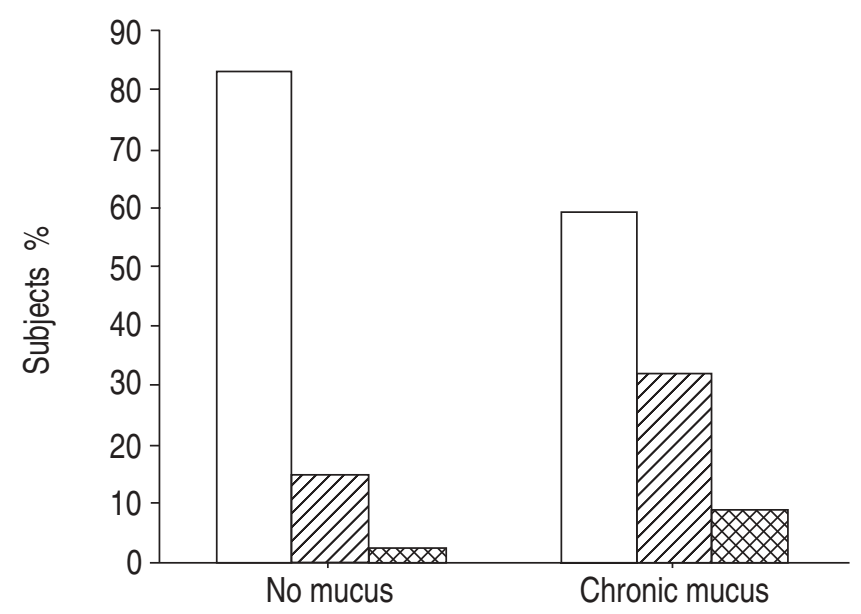

Fig. 1.-Chronic mucus hypersecretion as a predictor of respiratory infections. $\square$ : no episodes; $\mathbb{Z}: 1-5$ episodes; $\mathbf{2}: \geqslant 6$ episodes. lower in both sexes in subjects with $\mathrm{CMH}$, the decline of FEV1 appeared similar in those with and without CMH. These results differ slightly from the authors' previous observation in younger individuals in this cohort, where $\mathrm{CMH}$ was significantly related to accelerated FEV1 decline [4].

The importance of $\mathrm{CMH}$ as a predictor of survival and mortality from all causes and respiratory diseases was described using survival analysis. CMH was significantly associated with poor survival. When looking at specific causes of death using Cox regression models adjusting for age, sex and smoking, it was found that $\mathrm{CMH}$ was a significant predictor of death from obstructive lung disease (relative risk $(R R)=2.5$ ) and lung cancer $(\mathrm{RR}=2.0)$.

It is concluded that in the elderly population, $\mathrm{CMH}$ is a very common condition with important prognostic implications.

\section{Respiratory infections in the elderly}

Acute respiratory infections (ARI) are extremely common at all ages and in all countries. Most are mild, self-limiting illnesses and rarely fatal except in vulnerable groups such as the elderly and where social conditions are poor. Nevertheless, according to the Global Burden of Disease Study [5], in 1990 acute lower respiratory tract infections (LRTI) ranked third among the leading causes of death in the world, most of them $(75 \%)$ due to illnesses diagnosed as pneumonia. In contrast to mortality, age-specific incidence rates for ARI are similar in most communities regardless of differences in geography, and environmental and social conditions. However, the incidence of severe infections, like mortality rates, varies considerably by age and diagnosis. Thus, in persons aged $>65$ yrs, pneumonia and bronchitis are relatively more common than in younger adults [6].

There are several factors that may compromise the validity of statistics on mortality and morbidity. For example, there are no universally agreed clinical criteria for different ARI diagnoses. Consequently, there is wide variation in the use of the same diagnostic label by different practitioners [7]. Variable access to and use of microbiological laboratories, inadequate reporting systems, under-counting of ARI-related deaths arising from the application of coding rules that accord priority to the "underlying condition", and often uncertain population denominators, are further complicating factors. On balance, most published statistics probably greatly underestimate the true incidence of ARI illnesses and deaths.

\section{Acute lower respiratory illnesses}

Bronchitis and bacterial pneumonia occur in the elderly commonly as acute complications of chronic obstructive pulmonary disease (COPD) or secondary to viral infections. They also occur as terminal events, often due to nosocomial infection, for example with staphylococci, in patients who are seriously ill with other diseases.

Primary community-acquired pneumonia is the most common reason for acute admission to hospital in the UK [8]. Over $90 \%$ of these patients are aged $\geqslant 65 \mathrm{yrs}$, with a case fatality rate of $16-40 \%$ [9]. This age group is $2-5$ times more likely to develop pneumococcal infection than the general population [10], and fatality rates increase sharply with age. The most commonly identified pathogen is Streptococcus pneumoniae $(\leqslant 40 \%)$ [11], followed by Haemophilus influenzae and Staphylococcus aureus. Chlamydia pneumoniae has been reported in $\leqslant 26 \%$ of cases in patients $>65$ yrs [12]. "Atypical 
agents" collectively account for $10-20 \%$ of all cases of pneumonia [13]. They are becoming more prominent in older people, though some of the increase may be attributable to improved methods of detection [14].

\section{Chronic lower respiratory illness}

The role of infection in initiating damage to the lower airways and deterioration of respiratory function is uncertain. The healthy lower respiratory tract is normally sterile. There is evidence in animals that virus infections compromise the natural mechanisms for clearance of bacteria from the bronchi and enhance their invasiveness [15]. The possible role of primary bacterial infection is less clear.

One study found that carrier rates of $S$. pneumoniae and $H$. influenzae in the nasopharynx were similar for males with and without chronic sputum production [16]. This suggests that the presence of these organisms in the nasopharynx is not critical in initiating bronchial hypersecretion, although it may lead to secondary infection when other conditions favour invasion. The interpretation of cultures of expectorated sputum is confounded by possible contamination with organisms from the upper respiratory tract. However, one or both organisms was isolated much more frequently from sputum $(54 \%)$, particularly from purulent sputum $(75 \%)$, than from the upper respiratory tract $(25 \%)$ [16]. This suggests that in patients with mucus hypersecretion the bronchial tree is often colonised by bacteria, which then multiply during episodes of acute bronchitis.

The exact sequence of events leading to COPD remains uncertain, although FLETCHER et al. [17] were quite clear on the basis of their cohort studies that symptomatic bronchial infection was not a primary cause of COPD. While chronic subclinical infection might cause irreversible damage, FLETCHER et al. [17] thought this unlikely. On balance, it seems more likely that other agents, such as viruses, cigarette smoke and atmospheric pollutants, inflict the primary damage, which facilitates bacterial invasion leading to acute illnesses.

\section{Risk factors for respiratory infections}

Acute respiratory infections in early childhood. Exposure to several social and environmental factors, including indoor and outdoor air pollution, correlate with the incidence and severity of ARI. In children, local pollution exposure is important [18], while in adults smoking is the dominant risk factor. However, a residual effect of childhood respiratory illness and social conditions persists $[19,20]$. Recently, a strong correlation has been demonstrated between chronic bronchitis death rates in older adults and infant mortality rates from bronchitis and pneumonia some 50 yrs earlier [21]. Thus, the steep downward trend in bronchitis mortality in England and Wales during recent decades [22] may be linked to an earlier decline in LRTI in childhood [23], associated with improved environmental conditions. If the above relationships apply in newly emerging economies with increasing industrial pollution, there will be a substantial legacy of COPD to be reaped in later life [24].

Air pollution. Ambient air pollution and tobacco smoke can have short- as well as long-term adverse effects that lead to increased incidence and severity of respiratory infection. Experimental evidence in animals suggests that components of air pollution, such as ozone and nitrous oxide, impair clearance of bacteria from mouse lungs and increase fatality rates following acute infection. Also, exposure of macrophages to tobacco smoke reduces their ability to kill bacteria. However, these effects are not easy to demonstrate epidemiologically in human populations because of confounding with other environmental factors. The effects are most obvious during short episodes of intense exposure. In December 1991, for example, London experienced an historic air pollution episode lasting 4 days. During the episode, deaths attributed to respiratory infections were $23 \%$ higher than expected, consultations with general practitioners rose by $10 \%$ for upper respiratory and $4 \%$ for lower respiratory conditions, and hospital admissions of people $>65$ yrs rose by $19 \%$ [25].

Smoking. The adverse effects of tobacco smoke on lung health are too well known to require emphasis [26]. The increased susceptibility to acute and chronic infection among smokers was starkly reflected in cohort studies of mortality from COPD, pneumonia and tuberculosis [27].

\section{Prevention of acute respiratory infections}

The prevention of ARI and of its damaging consequences depends on reduction of exposure to aetiological agents, the control of factors that increase susceptibility to ARI, and on increasing immunity by vaccination.

Reduced exposure to potentially harmful microbes depends on the control of factors that favour cross-infection, such as poor housing, overcrowding and other adverse social conditions. Much more important would be reduced exposure to tobacco smoke and indoor air pollution from the combustion of fossil fuels in conditions with poor ventilation.

Presently, the available vaccines for the elderly include influenza and pneumococcal vaccines. Killed influenza virus vaccines are effective in preventing illness and reducing the frequency of complications in the elderly [28, 29]. Vaccination has also been shown to reduce hospital admissions and mortality [30] and save money [31]. Unfortunately, owing to antigenic shift and drift the composition of the vaccine has to be revised annually to include the most recent $\mathrm{A}$ and $\mathrm{B}$ variants, and annual re-vaccination with the current vaccine is essential to sustain protection. Many countries recommend routine immunisation of all persons aged $>65 \mathrm{yrs}$, in addition to high-risk groups such as those with chronic heart or lung disease.

Pneumococcal vaccine contains purified capsular polysaccharide from each of 23 capsular types of pneumococcus, which account for $\sim 90 \%$ of the pneumococcal isolates causing serious infection [32]. A single dose is usually recommended for patients at high risk from developing pneumococcal disease [33], including those with chronic heart and lung disease, while some authorities advocate immunisation of all persons aged $>65$ yrs [34]. Overall efficacy in preventing pneumococcal pneumonia is probably $\sim 60-70 \%$. Booster doses of vaccine can provoke serious reactions and are not normally advised within 5-10 yrs.

In conclusion, knowledge of the causes, consequences and prevention of ARI in the elderly has advanced little in the last two or three decades. Further research is needed into: 1) Improved data on mortality and morbidity attributed to ARI in different population groups; 2) simplified techniques for identifying aetiological agents; 3) mechanisms whereby the lower respiratory tract resists infection; 4) how lung defences may be damaged by exposure to tobacco smoke, atmospheric pollutants and infection; 5) the role of infection and other risk factors in childhood in the origins and progression of COPD in later life; and 6) evaluation of the efficacy and cost-effectiveness of vaccines for the prevention of respiratory illnesses and death in the elderly. 


\section{Cardiovascular comorbidity}

Cardiovascular comorbidity contributes significantly to the prognosis of elderly subjects affected by COPD. Much epidemiological evidence of the association between air pollution and increased rates of cardiovascular morbidity and mortality in elderly subjects has been produced in the last decade. Moreover, that association seems to be a cause/effect relationship according to some recently published papers. It seems that cardiovascular diseases are not only the most relevant comorbidity for patients affected by COPD, but the cardiovascular system may be directly damaged by the same environmental pollutants primarily affecting the respiratory system.

\section{The epidemiological evidence}

In a cohort of patients affected by COPD and respiratory failure, it was shown that the presence of ventricular or atrial arrhythmias was the most relevant comorbid factor, increasing the in-hospital mortality rate of these patients [35]. Also, the long-term mortality rate in the same cohort was increased by the presence of electrocardiographic signs of right ventricular hypertrophy and of previous myocardial infarction or ischaemia [36]. Moreover, some electrocardiographic patterns of right ventricular hypertrophy predicted a very poor prognosis and a median survival time no longer than 2 yrs [37]. In short, when bronchial obstruction in COPD patients reached its final stages, the pulmonary function parameters were no longer significant predictors of prognosis, and cardiovascular health seemed to be the main determinant of quality of life and survival.

The association of air pollution and mortality in the general population and, particularly, in the frail elderly population already affected by chronic pulmonary or cardiac diseases, has been demonstrated in a number of episodes, the most famous of which was the "London fog" in the winter of 1952. However, the main current concern derives from the demonstration that air pollution is a determinant of excess mortality at concentrations that are much lower than those recorded during the "London fog" episode, with a continuous linear association at concentrations considered safe by the regulatory authorities. Some recently published data [38], obtained from a large dataset including 20 US cities, confirm an association between fine particulate pollution and excess mortality, which is specific for that pollutant and, more relevant, for cardiovascular and respiratory cause-specific mortality rates. Not only mortality, but also morbidity from cardiovascular diseases is associated with air pollution. Some recently published papers report an association between the hospitalisation rate for cardiovascular diseases and air pollution [39-42]. Thus, there is consistent epidemiological evidence for an association between cardiovascular morbidity and mortality and fine particulate pollution. However, until recently, industry groups argued that the association found by epidemiologists could be attributable to residual confounding on the basis of the lack of biological plausibility for a causal relationship between pollutants and cardiovascular diseases [43].

\section{The biological plausibility}

Ongoing research is directed to two alternative ways of investigating a causal relationship between fine particulate pollution and cardiovascular disease: the "blood hypothesis" and the "autonomic hypothesis".
The "blood hypothesis" was advanced some years ago in a Lancet editorial [44]. The authors proposed that some biological mediators released from alveolar cells, stimulated by inhaled ultrafine particles could enhance blood coagulability, producing vascular thrombosis and cardiovascular damage. SEATON et al. [44] suggested that their hypothesis could be easily challenged, both in observational and experimental studies. Unfortunately, up to now, only a few inconsistent observational findings have been produced [45, 46], and the "blood hypothesis" is still an unproved, but fascinating hypothesis.

The "autonomic hypothesis" was suggested on the basis of some experimental results in dogs [47] and the observation of increased heart rate or ventricular arrhythmias associated with increased levels of fine or ultrafine particulates in the environment [48, 49]. More recently, the association between the autonomic control of the heart and air pollution has been investigated in observational studies that showed that decreased heart rate variability was found to be associated with increased levels of particulates $[50,51]$. These results and the well-known poor prognosis associated with decreased heart rate variability in subjects affected by ischaemic heart disease and other cardiovascular problems [52] strongly suggest that the linkage between cardiovascular morbidity and air pollution may be found in some disturbance of the autonomic control of heart activity.

In conclusion, cardiovascular diseases are the most important determinant of prognosis of elderly subjects affected by severe bronchial obstruction due to chronic obstructive pulmonary disease. Particulate air pollution increases the mortality and morbidity rates in elderly subjects, but the currently available epidemiological data are not suitable to further disentangle the sequence of events and the relative importance of pulmonary and cardiovascular problems that result in hospitalisation or death of those subjects. To resolve this problem, it is advisable to perform prospective cohort studies of subjects affected by pulmonary and cardiac diseases already defined with a high degree of accuracy and resident in areas with high-quality environmental monitoring facilities. These studies should aim to analyse the association of episodes of exacerbation and hospitalisation or death with air pollution levels in subjects with a well-defined clinical situation both before and after the critical episodes. The exact definition of "who" and "how many" individuals comprise susceptible subjects is the main anticipated result of this study, with obvious implications for setting new air quality standards.

\section{References}

1. Enright PL, Kronmal RA, Higgins MW, Schenker MB, Haponik EF. Prevalence and correlates of respiratory symptoms and disease in the elderly. Cardiovascular Health Study. Chest 1994; 106: 827-834.

2. Appleyard M, ed. The Copenhagen City Heart Study. Scand J Soc Med Suppl 1989; 41: 1-160.

3. Lange P, Groth S, Nyboe J, et al. Chronic obstructive lung disease in Copenhagen: cross-sectional epidemiological aspects. J Intern Med 1989; 226: 25-32.

4. Vestbo J, Prescott E, Lange P. Association of chronic mucus hypersecretion with FEV1 decline and chronic obstructive pulmonary disease morbidity. Copenhagen City Heart Study Group. Am J Respir Crit Care Med 1996; 153: 1530-1535.

5. Murray CJL, Lopez AD. Mortality by cause for eight regions of the world: Global Burden of Disease Study. Lancet 1997; 349: 1269-1276.

6. Miller DL. Collaborative studies of acute respiratory disease in patients seen in general practice and in children admitted 
to hospital: aims, field methods and morbidity rates. Postgrad Med J 1973; 49: 749-762.

7. Hope-Simpson RE, Miller DL. The definition of acute respiratory illnesses in general practice. Postgrad Med J 1973; 49: 763-770.

8. British Thoracic Society. Guidelines for the management of community-acquired pneumonia in adults admitted to hospital. Brit J Hosp Med 1993; 49: 346-350.

9. Woodhead MA. Pneumonia in the elderly. $J$ Antimicrob Chemother 1994; 34: Suppl. A, 85-92.

10. Sims RV, Boyko EJ, Maislin G, Lipsky BA, Sanford Schwarz J. The role of age in susceptibility to pneumococcal infection. Age Ageing 1992; 21: 357-361.

11. Farr BM, Kaiser DL, Harrison BD, Connolly CK. Prediction of microbial aetiology at admission to hospital for pneumonia from presenting clinical features. Thorax 1989; 44: 1031-1035.

12. Lieberman D, Schlaffer F, Porath A. Community-acquired pneumonia in old age. Age Ageing 1997; 26: 69-75.

13. Bartlett JG, Mundy LM. Community-acquired pneumonia. N Engl J Med 1995; 333: 1618-1624.

14. Wort SJ, Rogers TR. Community-acquired pneumonia in elderly people. $B M J 1998$; 316: 1690.

15. Miller DL. The role of infection and air pollution in chronic respiratory disease. In: Holland WW, ed. Air Pollution and Respiratory Disease. Westport, Technomatic, 1972; pp. 101-121.

16. Miller DL, Jones R. The bacterial flora of the upper respiratory tract and sputum of working men. J Path Bact 1964; 87: 182-186.

17. Fletcher C, Peto R, Tinker C, Speizer FE. The Natural History of Chronic Bronchitis and Emphysema. Oxford, Oxford University Press, 1976; pp. 133-135.

18. Douglas JWB, Waller RE. Air pollution and respiratory infection in children. Brit J Prev Soc Med 1966; 20: 1-8.

19. Kiernan KE, Colley JRT, Douglas JWB, Reid DD. Chronic cough in young adults in relation to smoking habits, childhood environment and chest illness. Respiration 1976; 33: 236-244.

20. Britten N, Davies JMC, Colley JRT. Early respiratory experience and subsequent cough and peak expiratory flow rate in 36-year-old men and women. Brit Med J 1987; 294 : 1317-1320.

21. Barker DJP, Osmond C. Childhood respiratory infection and adult chronic bronchitis in England and Wales. Brit Med $J$ 1986; 293: 1271-1275.

22. Colley JRT. Chronic non-specific lung disease (CNSLD) and asthma. In: Miller DL, Farmer RDT, eds. Epidemiology of Diseases. Oxford, Blackwell, 1982.

23. Waller RE. Atmospheric pollution. Chest 1989; 96: Suppl. 3, 363S-367S.

24. Colley JR, Miller DL. Acute respiratory infections. Chest 1989; 96: Suppl. 3, 355S-360S.

25. Macfarlane A, Haines A, Goubet S, Anderson R, Ponce de Leon A, Limb E, Air pollution, climate and health: shortterm effects and long-term prospects. In: Charlton J, Murphy M, eds. The Health of Adult Britain, 1841-1994. Vol. 1. London, The Stationery Office, 1997; pp. 187-204.

26. Doll R. Risk from tobacco and potentials for health gain. Int J Tuberc Lung Dis 1997; 3: 90-99.

27. Doll R, Peto R, Wheatley K, Gray R, Sutherland I. Mortality in relation to smoking: 40 years' observation on male British doctors. BMJ 1994; 309: 901-911.

28. National Health Service Centre for Reviews and Dissemination, The University of York. Influenza vaccination and older people. Effectiveness Matters 1996; 2 (issue 1).

29. Diguiseppi C. Why everyone over 65 deserves influenza vaccine. BMJ 1996; 313: 1162.

30. Department of Health. Influenza Immunisation, Annex 1. PL/CMO/2000/3, PL/CNO/2000/6, PL/CPHO/2000/1. London, Dept of Health.
31. Nichol KL, Iviargolis KL, Wuorenma J, Von Sternberg T. The efficacy and cost-effectiveness of vaccination against influenza among elderly persons living in the community. N Engl J Med 1994; 331: 778-784.

32. Johnson AP, Speller DCE, George RC, Warner M, Domingue G, Efrastiou A. Prevalence of antibiotic resistance and serotypes in pneumococci in England and Wales: results of observational surveys in 1990 and 1995. Brit Med J 1996; 312: 1454-1456.

33. Salisbury DM, Begg NT, eds. Immunisation Against Infectious Disease. Department of Health. London, HMSO, 1996; pp. 167-172.

34. Anonymous. The place of pneumococcal vaccination. Drug Ther Bull 1998; 36: 73-76.

35. Fuso L, Incalzi RA, Pistelli R, et al. Predicting mortality of patients hospitalized for acutely exacerbated chronic obstructive pulmonary disease. Am J Med 1995; 98: 272-277.

36. Antonelli Incalzi R, Fuso L, De Rosa M, et al. Co-morbidity contributes to predict mortality of patients with chronic obstructive pulmonary disease. Eur Respir J 1997; 10: 2794 2800.

37. Incalzi RA, Fuso L, De Rosa M, et al. Electrocardiographic signs of chronic cor pulmonale: A negative prognostic finding in chronic obstructive pulmonary disease. Circulation 1999; 99: 1600-1605.

38. Samet JM, Dominici F, Curriero FC, Coursac I, Zeger SL. Fine particulate air pollution and mortality in 20 U.S. cities, 1987-1994. N Engl J Med 2000; 343: 1742-1749.

39. Burnett RT, Dales R, Krewski D, Vincent R, Dann T, Brook JR. Associations between ambient particulate sulfate and admissions to Ontario hospitals for cardiac and respiratory diseases. Am J Epidemiol 1995; 142: 15-22.

40. Schwartz J, Morris R. Air pollution and hospital admissions for cardiovascular disease in Detroit, Michigan. $A m J$ Epidemiol 1995; 142: 23-35.

41. Poloniecki JD, Atkinson RW, de Leon AP, Anderson HR. Daily time series for cardiovascular hospital admissions and previous day's air pollution in London, UK. Occup Environ Med 1997; 54: 535-540.

42. Zanobetti A, Schwartz J, Dockery DW. Airborne particles are a risk factor for hospital admissions for heart and lung disease. Environ Health Perspect 2000; 108: 1071-1077.

43. Ware JH. Particulate air pollution and mortality - clearing the air. N Engl J Med 2000; 343: 1798-1799.

44. Seaton A, MacNee W, Donaldson K, Godden D. Particulate air pollution and acute health effects. Lancet 1995; 345 : 176-178.

45. Seaton A, Soutar A, Crawford V, et al. Particulate air pollution and the blood. Thorax 1999; 54: 1027-1032.

46. Pekkanen J, Brunner EJ, Anderson HR, Tiittanen P, Atkinson RW. Daily concentrations of air pollution and plasma fibrinogen in London. Occup Environ Med 2000; 57: 818-822.

47. Godleski JJ, Verrier RL, Koutrakis P, et al. Mechanisms of morbidity and mortality from exposure to ambient air particles. Res Rep Health Eff Inst 2000; 91: 5-88.

48. Peters A, Perz S, Doring A, Stieber J, Koenig W, Wichmann HE. Increases in heart rate during an air pollution episode. Am J Epidemiol 1999; 150: 1094-1098.

49. Peters A, Liu E, Verrier RL, et al. Air pollution and incidence of cardiac arrhythmia. Epidemiology 2000; 11: 11-17.

50. Pope CA III, Verrier RL, Lovett EG, et al. Heart rate variability associated with particulate air pollution. Am Heart J 1999; 138: 890-899.

51. Gold DR, Litonjua A, Schwartz J, et al. Ambient pollution and heart rate variability. Circulation 2000; 101: 1267-1273.

52. Lombardi F. Chaos theory, heart rate variability, and arrhythmic mortality. Circulation 2000; 101: 8-10. 